\title{
User Experiences with Mobile Phone Camera Game Interfaces
}

\author{
Sam Bucolo \\ School of Design \\ Queensland University of Technology \\ +61 738642076 \\ s.bucolo@qut.edu.au
}

\author{
Mark Billinghurst \\ HIT Lab NZ \\ University of Canterbury \\ +6433642349 \\ mark.billinghurst@hitlabnz.org \\ David Sickinger \\ HIT Lab NZ \\ University of Canterbury \\ +64 $33642987 \times 3078$ \\ david.sickinger@hitlabnz.org
}

\begin{abstract}
This paper presents the findings of a comparative study investigating different input interfaces for a mobile phone games application. A standard mobile phone joystick interface is compared with a phone camera interface to detect the phone translation and tilt to control a ball's movement within various levels of difficulty of a virtual maze game. Game completion times together with the resultant user experience for each of the games was collected during the studies. Results indicate that the joystick control provided the fastest completion times for each game, but with the lowest levels of user engagement. The Tilt interface, although perceived as challenging by the participants, provided the greatest level of user involvement, independent of game complexity. The design of appropriate human interfaces which go beyond the standard phone keypad is suggested. The selection and design of these interfaces should also consider the intended user experience for the game.
\end{abstract}

\section{Categories and Subject Descriptors}

H.5 [Information Interfaces and Presentation]: Multimedia Information Systems

\section{General Terms}

Human Factors, Experimentation and Design

\section{Keywords}

Mobile Camera Phones, Mobile Games, Human Interaction, Tangible Interfaces, Novel Interfaces.

\section{INTRODUCTION}

In this paper we present a study which has developed and compares three human interfaces appropriate for a mobile phone game application. A key challenge in developing a successful mobile game is the constraints imposed by the device itself. Game developers need to take into consideration screen resolution, processor constraints, memory restrictions and download options. In addition to this the game developer is generally also limited to the 12 button keypad configuration as the primary human interface for their game. In this work we report on the development of games that use input from the camera on a mobile phone. We show how computer vision applications can be developed which run entirely on the phone, enabling new types of handheld game interactions.

\section{MOBILE GAMES MARKET}

Over the past five years mobile phones have become a ubiquitous communications device. Current mobile phones have fast processors (100+ Mhz), sharp colour screens, and multimedia functionality. So it is little surprise that there is a rapidly growing market for games for mobile phones.

While it is relatively easy to port classic computer games over to the mobile phone, there is also an opportunity to develop very new types of games that take advantage of the unique functionality provided by the mobile phone. Cameras are becoming ubiquitous on mobile phones and so provide an obvious input device for novel games. There will be more cameras in mobile phones than in any other device by 2010, making it the ideal platform for vision-based applications. There is also incentive for the service providers to support such applications. With the introduction of 3rd Generation (3G) networks, providers are looking for applications that use more bandwidth. Games that involve image and data sharing are ideal.

In this paper we report on a novel computer vision based game we have developed for mobile phones and present a user evaluation that compares user input with the camera to more traditional keypad input. This is one of the first papers that presents a user study of a computer vision based mobile phone game.

\section{RELATED RESEARCH}

Although there are thousands of games available for mobile phones, there are only a handful of games that use associated sensors and very few that use the phone's camera for game input.

The main ways that camera input has been used in existing games is to provide simple phone position and orientation tracking. For example, the "Mosquito Hunt" [7] and "Marble Revolution" [4] games both use simple optical flow techniques to track the phone motion. In the "Mosquito Hunt", virtual mosquitoes are superimposed over the real world image from the camera. As the user moves the phone they can position a virtual crosshair relative to the live video background on the screen and shoot the 
mosquitoes. In the "Marble Revolution" game, either the joystick or input from the phone camera can be used to steer a marble through a maze. In this case motion flow techniques are used to estimate the tilt of the phone relative to the real world scene and so provide input to steer the marble. Using the camera mode just requires slight movement of the phone to mimic the feel of the old wooden tablet games. The "SymBall" table tennis game [10] is a little more complex. Here a player selects a primary colour (red, blue or green) and blob tracking is used to track the phone position relative to the largest patch of this colour. This is then used to control the position of a tables tennis paddle on the camera screen in a virtual table tennis game.

There are also more complex computer vision examples that use the phone for image capture, but process the images off the phone before sending the output back to the handheld display. But the downside to these types of systems is the considerable lag time before results appear on the phone screen, making this approach inappropriate for real time gaming.

Our work is also related to research in novel input techniques for handheld devices. Unlike computer interfaces, handheld devices lend themselves to physical manipulation. Beverley et. al. [1998] showed that physical interaction such as squeezing and tilting could be naturally supported by adding physical sensors to a handheld computer. Fitzmaurice [1993], and Rekimoto [1996] also explore the use of additional sensors to small displays for detecting tilt or position. In this way the motion of the displays can be used to display data from a larger surrounding virtual information space. Hinckley et. al. [2000] describe in more general terms how a variety of different sensors can be added to mobile devices to enable applications to respond in a natural manner to the behavior of the device. For example the device turns itself on when it is picked up and changes the screen information display depending on its physical orientation. In these examples researchers added sensors to the device to provide additional input capability. In our work we want to support physical based interaction from camera input alone, developing novel games that don't require the use of any additional hardware.

Augmented Reality (AR) interfaces also often involve the use of cameras to superimpose virtual images over live video of the real world. Applications such as AR Quake [Piekarski el al 2002] and Shared Space [Billinghurst et al 2000] show how compelling AR gaming can be. However AR games have typically been played with head mounted displays. The modern phone provides a camera, display, significant processing power and a graphics engine all suitable for running AR applications in a handheld configuration. Moehring et al. [2004] have already demonstrated the first AR interfaces running on a consumer mobile phone, while Mogilev et. al [2002] provide an example of a handheld AR game that could be suitable for the mobile phone platform.

In order to explore camera-based input for mobile phones there are a number of computer-vision libraries that can be used. For example, the SpotCode [6] image processing library uses special round markers that can be recognized by a mobile phone and the orientation, position, and size can be calculated. The Phone Vision Library [5] is a generic computer vision library for use with mobile phones. The popular ARToolKit [3] augmented reality tracking library has also been ported onto a phone application.
Although the current camera-based games are very interesting there has been no published research on the usability of camerabased game input on a mobile phone or the user response to these types of games. To investigate the potential of camera driven input a mobile game application was developed incorporating camera tracking as the interface for the game, thereby eliminating the need for a keypad. In the next section we outline the game we developed and in sections five and six provide evaluation results.

\section{GAME DESIGN}

To explore camera-based input we have developed a Mobile Maze game for the mobile phone. The Mobile Maze is based on the traditional game where the objective is for the user to control the movement of a ball through a physical maze by tilting the maze by hand (Figure 1). The innovation in our game lies in the development of an emerging paradigm for user interaction with mobile devices. The game extends the keypad paradigm for game interaction through the use of a mobile phone camera to provide tracking of user movements, thereby creating a tangible user interface for mobile devices (Figure 2). It is the combination of this simple game format and advanced interaction paradigm which aims to provide an engaging user experience and innovative mobile game concept / platform.

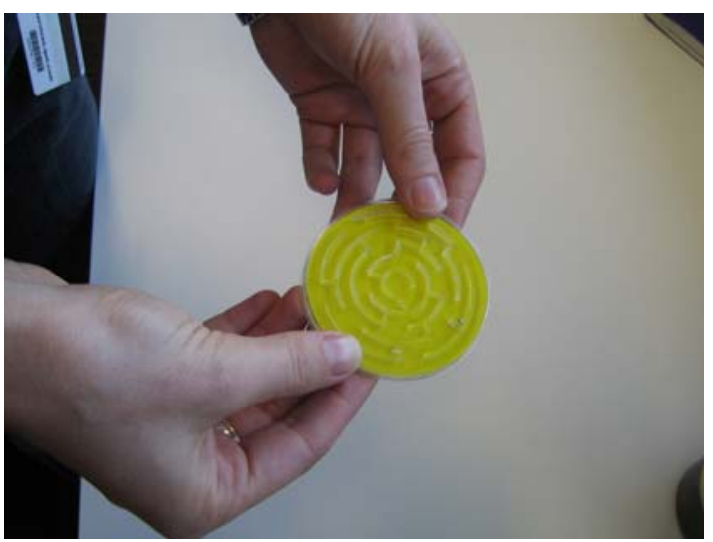

Figure 1. Mobile Maze Game Analogy.

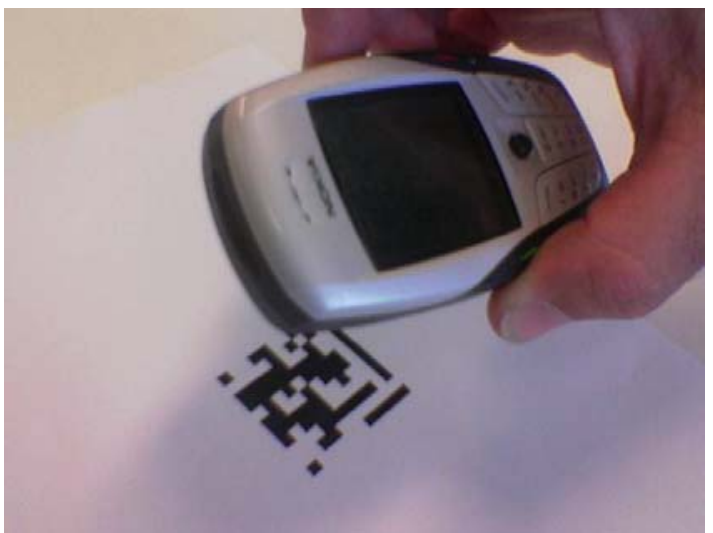

Figure 2. Mobile Maze Game and Markers

\subsection{Technical Specification}

The Mobile Maze application was written in $\mathrm{C}++$ for the Symbian OS using the Series 60 (v2.1) SDK platform. The Nokia 6600 
mobile phone was used during development and for the user testing. This device contains a $104 \mathrm{MHz}$ ARM-9 series CPU, 6 MB of memory, a $176 \times 208$ pixel sized screen, and a 0.3 megapixel camera.

The marker system used in the study game testing application was the Visual Codes for Symbian OS [8]. This visual code system allows camera-equipped mobile phones to recognize special 2-D markers and it computes the phone's orientation relative to a coded marker. The phones tilt and translation are among the values computed by the Visual Codes system.

In the prototype game application two phone camera interfaces were compared against traditional joystick input. Depending on the phone camera interface method selected, the tilt values or translation values (each have horizontal and vertical components) were compared against threshold values to determine if an attempt to move the ball should happen and in what direction. If the tilt values or translation values fall within the thresholds, then no movement occurs during that update call. If the joystick interface is selected during testing, the camera still takes an image but the joystick directional input is used instead.

Once the ball is moving, an important part of the application is checking for collisions between the ball and the maze. The wall collision check compares the ball's intended new center position against a mask image of the maze. The mask image is made by thickening the walls of the maze to the width of the radius of the ball. In order to make the mazes complex enough, the ball diameter had to be kept small to allow more possible paths in the maze (a decision was made not to have scrolling mazes). The minimum wall thickness of the mask images was 6 pixels which meant the ball could not move by more than this amount without risking the ball moving through walls. So the ball movement was set at two pixels per update.

A splash screen pops up when the game is first launched and the user is prompted to press the Left Selection Key to access a menu of input options. The three choices are:

1) The user could use the phone's joystick

2) Using the camera on the phone to detect the phone tilt

3) Using the camera to detect the phone translation (panning)

A Visual Code marker is shown in Figure 2. If one of the camera interfaces is selected, then the user must position the phone so the marker is in the field-of-view of the camera to update the ball movement. If a marker is not detected during an update step, then the direction from the last ball update is used as input. In the future the use of camera motion flow tracking techniques will replace the need for using markers.

The elapsed game time is displayed in the upper left hand corner of the screen. A simple scoring system was implemented for the study game testing. The number of seconds that has passed since starting the game is subtracted from an initial score of 1000 .

\subsection{User Study Design}

The focus of the user study was to compare performance with the three different input options mentioned above.

A submenu prompting the user to select one of three studies is displayed when the user presses Select Study on the main option screen. If Study 1 is selected, then another submenu is displayed prompting the selection between three moderate games. Screen shots of the mazes used for the moderate level complexity games are shown in Figure 3. Game 1-A uses the tilt technique to control the ball movement, Game 1-B uses the pan technique, and Game 1-C makes use of the joystick control. A similar submenu is displayed when Study 2 (Figure 4) is selected and the user is prompted to select a complex game either Game 2-A, Game 2-B, or Game 2-C.

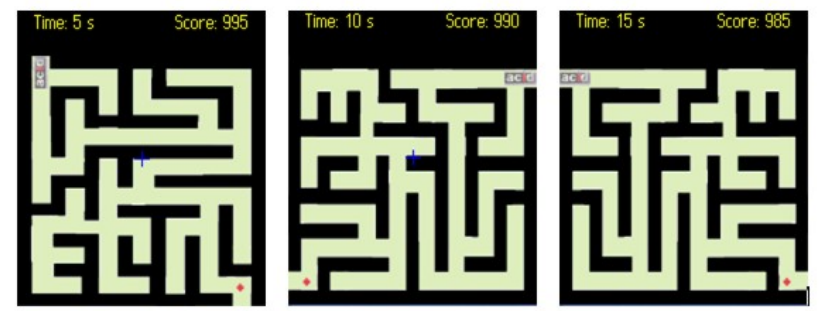

Figure 3. Moderate Game Mazes

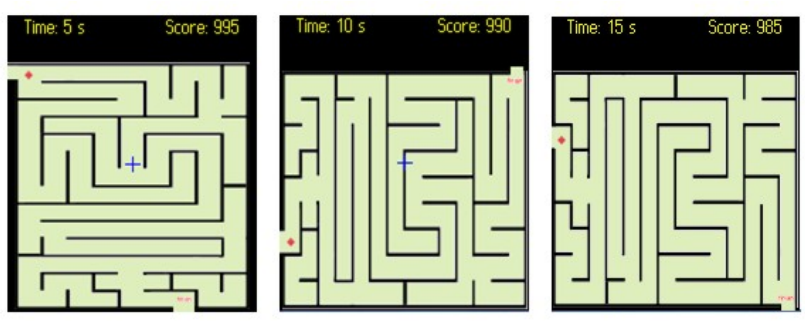

Figure 4. Complex Game Mazes

The mazes were made to be very similar to one another, with the same maze just rotated or mirrored vertically for each of the user trials. The thin walls used for the complex maze series allowed more pathways to be included making it slightly more difficult to solve than the thicker walled moderate series.

If a user selects Study 3 in the menu, the maze that is used is determined by the visual marker that the phone camera first detects. The user is first prompted to select either the moderate (equivalent to Study 1) or complex (equivalent to Study 2) level of maze difficulty. Then the user can point the camera at a marker numbered one to three to select what maze will appear (Figure 5).

Study 3 was not used in the usability test because of the need for an additional step of using a marker to select the maze. However, Study 3 did prove useful for allowing the user to practice where to hold the phone's camera relative to the marker to achieve the desired motion. Text output is displayed when this application is running indicating what direction the ball would move based on the camera's position if the camera is pointed at any Visual Code marker that is not numbered one to three. This allows the user to get familiar with the tilt and pan techniques.

\section{Mobile Game Evaluation}

The field of mobile application development is an emerging area of research. When evaluating mobile games applications, consideration of the intended user experience is paramount. 


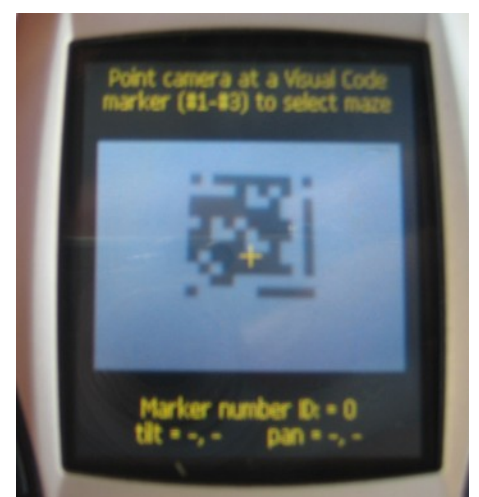

Figure 5: Selecting the Maze using the Visual Code

Bendas [2002] notes that for a mobile game to be commercially profitable, it should feature a set of characteristics such as playability and interoperability that are often conflicting or hard to reach. In evaluating mobile games, technical constraints should be matched with the intended user experience. This approach was applied to the evaluation of the Mobile Maze Game.

\subsection{Mobile Maze - Comparative Study}

A comparative study was undertaken which evaluated users experience and interaction with each of the three input approaches both within a moderate and complex game environment. Eleven participants from the university volunteered for the study.

The study group consisted of 6 male and 5 females of whom 7 were right handed and 4 left handed. The majority of the participants were in the 18-25 years age bracket. Most participants had prior games experience (primarily PC and console games). All but one participant owned their own mobile phone, with the greatest use of this device to make voice calls or SMS communication. The majority of participants had no experience with mobile game applications.

Prior to beginning the study, participants were asked to complete an ethics consent form and a questionnaire indicating their PC, Console and mobile game experience. Following this participants were provided with an overview of the types of game inputs (Tilt, Pan and Joystick) and were given the opportunity of trialing their use. Participants generally required no more than a few minutes to be comfortable with the approaches.

Participants were then asked to complete six tasks as part of the study. Study 1A (Tilt), 1B (Pan) and 1C (Joystick) focused on moderate maze games, whereas Study 2A (Tilt), 2B (Pan) and 2C (Joystick) focused on the complex mazes. All participants were to complete all the tasks, but the task and study order was varied for each to prevent order effects occurring.

For each condition the following types of data was collected;

- Think aloud spoken comments

- The way the user held the phone for each game type

- The time to complete each maze

- An interview undertaken at the end of each study to determine the overall user experience for each game
Participants were encouraged to speak aloud and were informed that observations were being made during each study by the researcher who was seated next to them (Figure 6). The participants were also informed that their audio transcripts were also being recorded for possible future analysis. Following each type of study (moderate or complex) the participants were also asked to reflect on their user experiences for each game. The total study time was approximately 30 minutes per participant.

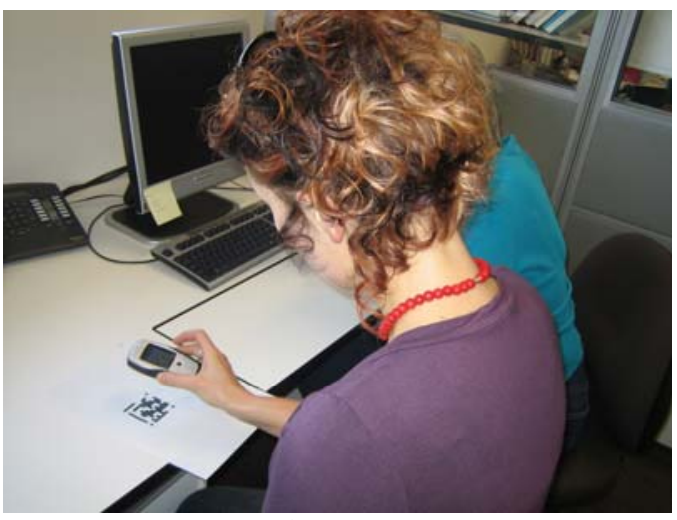

Figure 6. Study Environment

\section{Results / Findings}

\subsection{Holding Position}

The way the user held the phone was recorded to identify if the participants preferred to interact with the game using a position similar to that of taking a picture with a mobile phone (single handed vertical position) or using a standard two handed console position (two handed horizontal position - Figure 7).
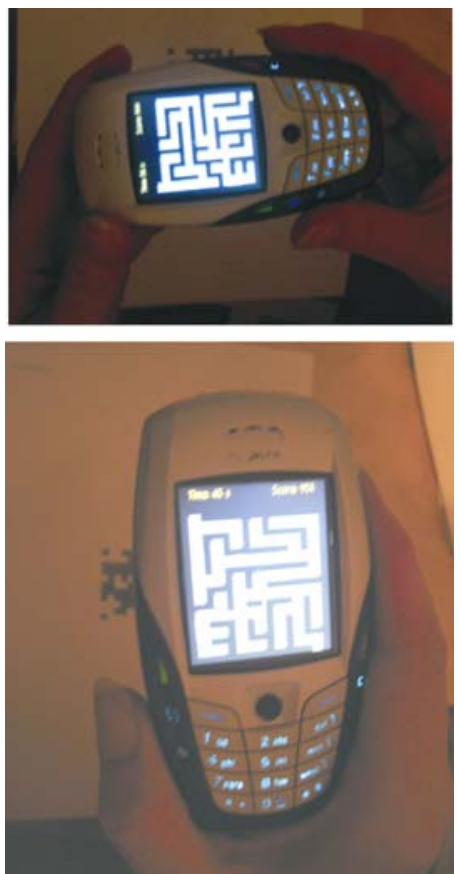

Figure 7: Phone Holding Positions 
Users had the choice of selecting their preferred position, with the only constraint being that the phone position must align with the marker, For example, if the camera was rotated 90 degrees into a horizontal position, the marker also required rotation.

As shown in figure 8, user preference was to hold the phone in a vertical position, using either one or two hands during each of the studies. Throughout all of the studies only one participant tried the horizontal holding position (study 2B) but then swapped back to a vertical position.

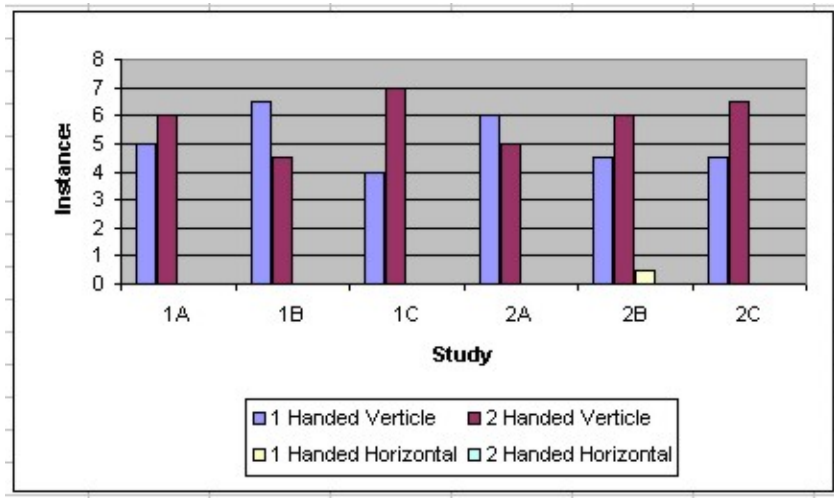

Figure 8: Phone Holding Positions

It was initially considered that the hand holding position would be similar to that of holding a physical maze game where the users hold the game with two hands in order to achieve maximum stability. It was also initially suggested that the participants would prefer to hold the phone in a horizontal position to mimic a console game and move away from focusing on the keypad.

A possible reason for this finding was that the game did not match the dynamics of a physical mouse game and therefore the two handed vertical control was needed to provide maximum control within the game. The vertical orientation also matched the affordances of the real phone design.

\subsection{Completion Time}

Completion time was recorded for each study to determine if the game interface contributed to delays in game play and if this was altered with varying levels of game complexity.

As can be seen in the following graphs (Figure 9), the joystick control was the most efficient interface for all participants. The average time for completion using the joystick for Study 1C was 55 seconds and for Study 2C, 95 seconds. All participants also completed the game using this interface.

Using the Tilt interface, completion times for Study 1A was higher than that for the joystick control. When used in Study 2A, three participants were unable to complete the maze using the Tilt interface. Of the participants who completed the game there was a considerable difference in the completion times.

The Panning interface was the most difficult to use. In Study 1C, seven participants failed to complete the maze. Of the participants who did finish the game the completion times were significantly higher than that for the joystick and tilt mode. In
Study 2C, several participants failed to complete the maze (6 of these were the same participants who were unable to complete the maze in Study 1). Again completion times were much higher than the other modes.

\subsection{In Game User Comments}

User comments were recorded to gain an understanding of the resultant user experience for the game when applying different input interfaces. They provide a further insight into the impact of the interface type within a mobile game context. The comments were recorded by the researcher during each study and if required were referenced with the audio transcripts for each study.

In general users commented that for both the moderate maze (Study 1C) and the complex maze (Study 2C) the joystick interface was far easier to use than the computer vision input. They felt that they could have good control over the ball motion and easily stop the ball when required. For example one user wrote "The focus is more on completing the game than trying to get the ball moving". However several subjects commented that there was no real challenge with the joystick and that it didn't offer anything new over existing games. One user wrote that "It can be boring because there is no challenge".

When using the pan input method, a common response from the users was that it was frustrating to control the movement of the ball both in the moderate (Study 2A) and complex configurations (Study 2B). Users were confused with the movement of the ball in relation to the movement of the phone. One user commented that "Thinking that up is down and down is up and another commented that "Controls are opposite to what they should be". Another issue raised related to the level of difficulty and the impact on the game play. A user commented that the complexity game was "much more difficult because of harder maze and imprecise controls".

The tilt input mode provided the greatest number of positive comments from the users. Although there was still an element of frustration in the control of the ball, users felt that the interface provided the greatest level of game challenge. The frustrations seem to come from the slow movement of the ball. One user commented that the tilt input mode "could be more sensitive to movement". When moving from the moderate (Study 1A) to the complex game (Study 1C), users noted on the increases level of difficulty, Only one user comment that this game (Study 1B) was easier with practice than the others.

A response common to both the title and pan input modes was the frustration on focusing the marker in order to play the game correctly. One user commented that they were "focusing more on getting the phone over the code (marker) than on getting the ball through the maze." Ensuring that the marker was visible seemed a difficult task in both game types. Users commented that they accidentally often put their hands over the camera or were unaware that the marker was not in view.

\subsection{Participant Reflections}

The final measure for the study was asking the participants to reflect on each overall study (Moderate or Complex Game). Participants were asked to comment on "the interfaces and their 


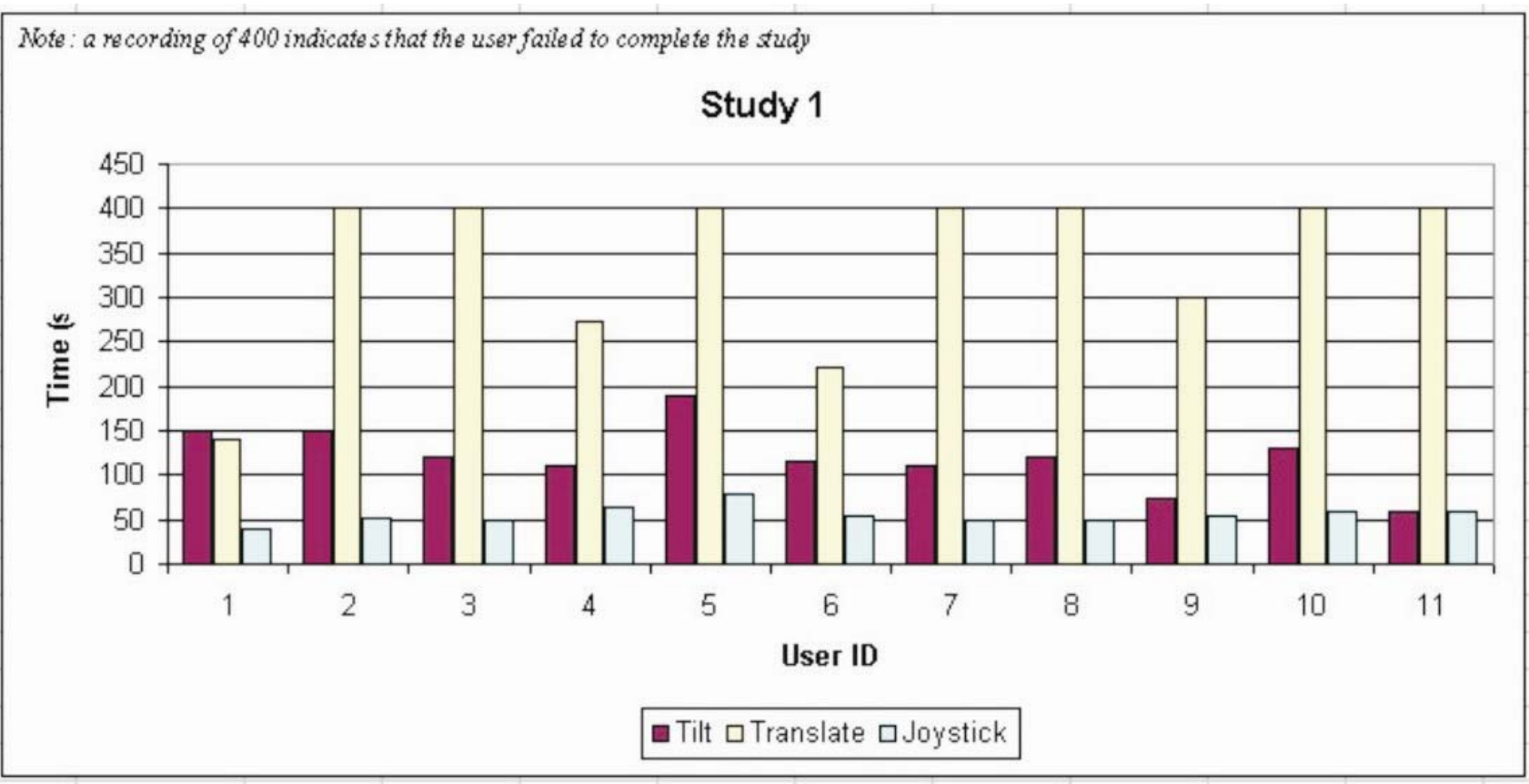

Note: a recording of 400 indicates that the user failed to complete the sudy

\section{Study 2}

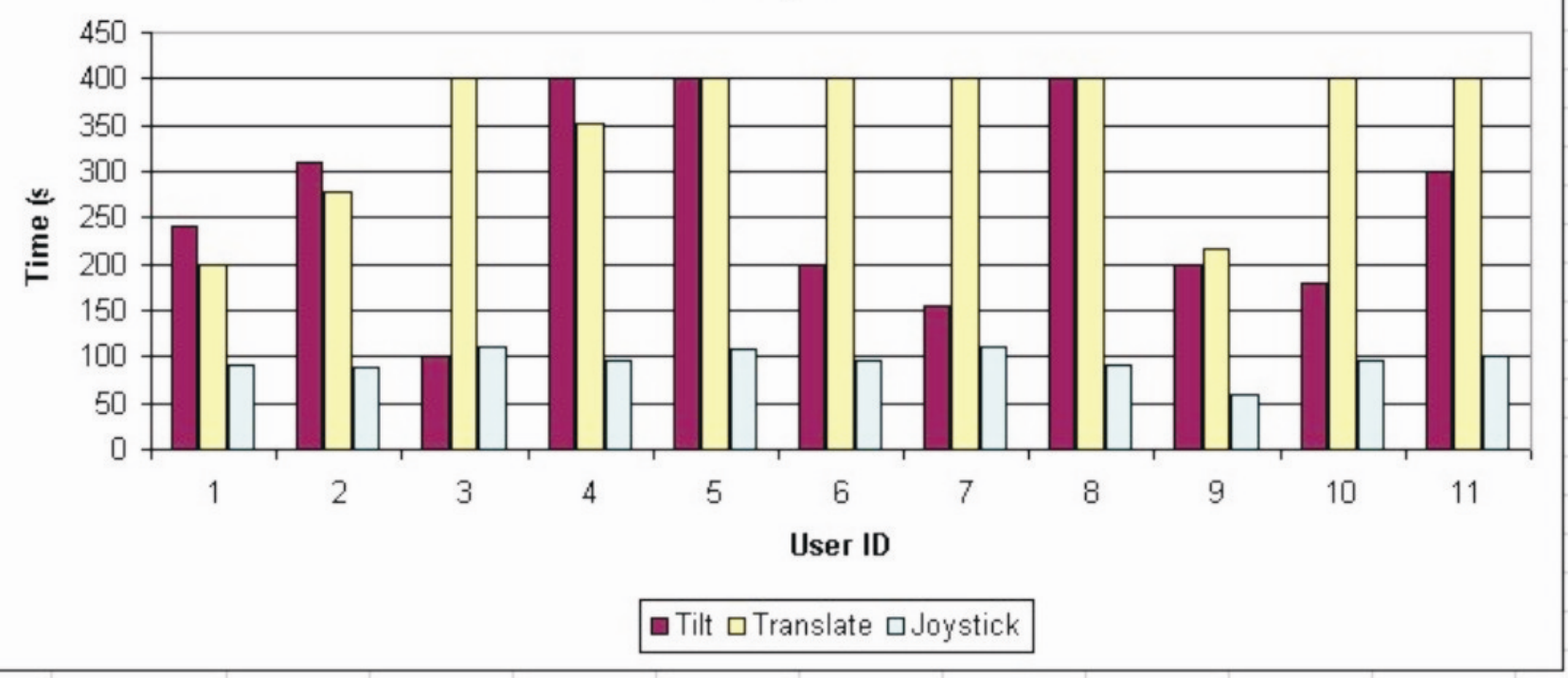

Figure 9: Completion Times

impact on the over game experience”. This was asked at the end of the moderate and complex studies. The recorded comments had greater depth in their responses and provided a useful mechanism to cross reference the In Game Recorded Comments.

In general for the tilt input mode in the moderate complexity (Study 1) users commented on how this mode provided the most enjoyable game challenge and enhanced their experience. One user commented on how tilting was easier, better and another felt that this mode would scale to a bigger game. One user

commented on their initial perception of the game, thinking panning would be easier, but it was not. Overall most users felt that although the tilt mode was difficult in the moderate configuration, it was an innovative use of the technology (still limited by technology but it is innovative). 
Where as in the complex configuration (Study 2), users commented that the mazes did provide a greater challenge but the

interface modes made it difficult and frustrating to complete. Summaries from the users included: Liked tilting, reminds me of pinball, but hard because you can not stop; Panning too hard, did not like the opposite movements. Joystick too easy.

\section{Discussion}

Before undertaking the study the authors anticipated that of the two computer vision input techniques the Panning input method would be a superior interface for the mobile maze game. Our assumption was based on the facts that as you tilted the camera, the user would not be able to view the screen and therefore would be unable to complete the game. However as indicated in the findings, the panning method was not favored by the study participants, who preferred the tilting interface for the game in both the moderate and complex game setup. Based on participant comments the panning method did not seem intuitive for the game. Comments such as Controls are opposite to what they should be and ...Thinking that up is down and down is up were commonly observed.

Although the Tilt interface was preferred it also had limitations such as ball speed and difficulty in seeing the marker to control the movement. However comments such as frustrating yet challenging were encouraging and suggested this interface to be appropriate to such a mobile games application. This findings relates to the fact that this interface mode matches the movement of a physical ball in a maze game and the users were comfortable with the transferring this to a virtual maze game.

A second assumption by the authors before the game began was that the players would hold the phone in a manner similar to that of hold a physical ball in a maze game, specifically in the two handed horizontal position to obtain maximum balance. As noted in the results, only one participant initially tried this position and then moved back to the vertical position. Suggested reasons for this relate to users being more comfortable with the vertical position of a phone as this is the orientation for most functions of the phone. A further reason relates to the speed of the game which did not match the dynamics and physics of a physical ball in a maze game and therefore the participants did not need to compensate for over tilting the maze using two hands. The issue of speed is being addressed in future versions of the game.

The use of time as a metric in evaluating the game also identified an interesting finding. The joystick mode recorded the quickest time in both the moderate and complex game configurations and participants quickly understood its use. However the recorded comments indicated that the final user experience from this mode was unsatisfactory eg can be boring because there is no challenge. As noted earlier, understanding the motivations and experience for game play is essential when considering usability evaluations. This finding highlights how a quantifiable metric such as time can easily be misinterpreted within such an evaluation. The rich data extracted from the user comments were an essential element of this study as it provided an insight into the participants experience with the interface within a game context.
Learning through repeated exposure of a particular interface mode was also highlighted within the study findings. A number of participants indicated within the complex game configuration (both tilt and panning) that they perceived the game and its control to be easier than the moderate game. This indicates that training of the particular interface may be necessary within a mobile games context for successful implementation of that interface. This could be incorporated as a separate module (as shown in study 3 ) or be incorporated into the game play.

\section{Design Recommendations}

The results of this experiment and the user feedback suggest several design recommendations that may be useful for other mobile games that use the phone camera phone and computer vision techniques to determine the motion of the phone.

1/ Provide feedback to the user when the camera is tracking the environment, especially if the camera image is not being shown on the screen. In our case we provided a yellow square that showed when the tracking marker was in the camera field of view.

2/ Games should be designed by taking into account the physical affordances of the phone. In this case the way users held the phone was largely due to the shape of the phone.

3/ Use the camera to calculate relative input rather than absolute input.

4/ Design games that do not rely on high speed camera input or rapid response to user motion.

5/ Design games which map natural interaction styles to that of the intended game play.

6/ Design games which do not require precise movement and control.

\section{Conclusions / Future Research}

This paper has presented a comparative study for a new type of interface in a mobile gaming application using the integrated camera commonly found in mobile phones. The game concept relates to a traditional game approach (ball in a maze type game) and the initial assumption from playing this type of game drove the development for this mobile game implementation. In particular the perceived final user experience from the traditional form of game was considered a benchmark for the mobile phone version.

The comparative study which was undertaken indicated the limitations with the current implementation of the game, in particular slow response time for the game and how the game did not match the performance or dynamics of the traditional type of game. The study suggests that the Tilt interface is the preferred method of interaction for such a game as it maintained the participant's interest as it provided a suitable challenge. The Panning mode was not preferred by the participants as it was perceived to be counter intuitive in terms of movement of the virtual ball. The Joystick mode was also not preferred as it provided the least challenge, however participants were able to complete the game in the least amount of time. This finding highlights the needs to consider the final user experience in the evaluation of mobile games. Relying on quantifiable metrics such as time can be misleading within such a study. 
Further research by the research group will focus on refinement and optimization of the game to take into consideration the comments by the participants of this study.

This is the first of a series of mobile phones games that we plan on developing in which camera input is used to determine physical phone motion. Exploring alternative game approaches using these interface types will also be explored and evaluated.

\section{ACKNOWLEDGMENTS}

The Authors would also like to acknowledge the funding support of the Australasian CRC for Interaction Design (ACID) and the Cooperative Research Centre Program through the Australian Government's Department of Education (http://www.interactiondesign.com.au/)

\section{REFERENCES}

[1] Assad, M., Cutting, D., and Hudson, A. AR phone: Accessible Augmented Reality in the Intelligent Environment. May 2004. http://www.smartinternet.com.au/SITWEB/publication/files/7 4_\$\$\$99243/P04_044.pdf

[2] Bendas D (2002), Games as Part of Mobile Entertainment, Proceedings of PROFES 2002, Finland

[3] ARToolKit on Symbian; www.itn.liu.se/ andhe/UMAR

[4] Marble Revolution; http://mysymbian.com/7650/applications/applications.php?fldAuto $=1$ $046 \& f a q=3$

[5] Phone Vision Library (PVL) R1.0; $\quad$ www.uniweimar.de/ bimber/research.php

[6] SpotCode; www.highenergymagic.com

[7] Virtual Mosquito Hunt: An Augmented Reality Game; http://w4.siemens.de/en2/html/press/newsdesk archive/2003/ e_0311_d.html\#Meldung1

[8] Visual Codes for Symbian OS; www2.inf.ethz.ch/ rohs/visualcodes
[9] Moehring, M., Lessig, C. and Bimber, O. Video SeeThrough AR on Consumer Cell Phones. In proceedings of International Symposium on Augmented and Mixed Reality (ISMAR'04), pp. 252-253, 2004

[10] Symball download website: http://www.vtt.fi/multimedia/download_symball.html

[11] Wayne Piekarski and Bruce Thomas, ARQuake: The Outdoor Augmented Reality Gaming System, Communications of the ACM,2002 Vol 45. No 1, pp 36-38

[12] Mogilev, D., Kiyokawa, K., Billinghurst, M., Pair, J. AR Pad: An Interface for Face-to-Face Collaboration. In Proceedings of CHI 2002. April 20-25 ${ }^{\text {th }}$, Minneapolis, Minnesota, 2002.

[13] Billinghurst, M., Poupyrev, I., Kato, H., May, R. Mixing Realities in Shared Space: An Augmented Reality Interface for Collaborative Computing. In Proceedings of the IEEE International Conference on Multimedia and Expo (ICME2000), July 30th - August 2, New York

[14] Beverly L. Harrison, Kenneth P. Fishkin, Anuj Gujar, Carlos Mochon, and Roy Want, Squeeze Me, Hold Me, Tilt Me! An Exploration of Manipulative User Interfaces. Proceedings of SIGCHI '98, (Los Angeles, CA, April 18-23) ACM, New York, 1998, pp. 17-24.

[15] Rekimoto, J. Tilting Operations for Small Screen Interfaces. Proceedings of UIST '96. pp.167-168.

[16] Fitzmaurice, G. Situated Information Spaces and Spatially Aware Palmtop Computers, CACM, Vol. 36, 7, July 1993, pp.38-49.

[17] Hinckley, K., Pierce, J., Sinclair, M., Horvitz, E., Sensing Techniques for Mobile Interaction, ACM UIST 2000 Symposium on User Interface Software \& Technology, CHI Letters 2 (2), pp. 91-100. 